SUBJECT AREAS:

FLUORESCENT DYES

NUCLEIC ACIDS

Received

25 November 2013

Accepted

23 December 2013

Published

20 January 2014

Correspondence and requests for materials should be addressed to Y.-T.C. (chmcył@nus. edu.sg)

* These authors contributed equally to this work.

\section{Discovery of a Structural-Element Specific G-Quadruplex "Light-Up" Probe}

\author{
Liyun Zhang ${ }^{1,2 *}$, Jun Cheng Err,3*, Krishna Kanta Ghosh' ${ }^{1}$, Wan Jun Chung ${ }^{4}$, Jaeduk Yoo' ${ }^{1}$ Wang Xu', \\ Wei Zhao ${ }^{5}$, Anh Tuân Phan ${ }^{4} \&$ Young-Tae Chang ${ }^{1,6}$
}

\begin{abstract}
'Department of Chemistry, National University of Singapore, 3 Science Drive 2117543 , Singapore, ${ }^{2}$ Hefei Institutes of Physical Science, Chinese Academy of Sciences, Hefei, Anhui 23003 1, P. R. China, ${ }^{3}$ Graduate School for Integrative Sciences and Engineering, National University of Singapore, Centre for Life Sciences, \#05-01, 28 Medical Drive, 117456 Singapore, ${ }^{4}$ Division of Physics and Applied Physics, School of Physical and Mathematical Sciences, Nanyang Technological University, Singapore 637371, Singapore, ${ }^{5}$ School of Life Sciences, University of Science and Technology of China, Hefei, Anhui 230027, P. R. China, ${ }^{6}$ Singapore Bioimaging Consortium, Agency for Science, Technology and Research (A*STAR), 138667, Singapore.
\end{abstract}

The development of a fluorescent probe capable of detecting and distinguishing the wide diversity of G-quadruplex structures is particularly challenging. Herein, we report a novel BODIPY-based fluorescent sensor (GQR) that shows unprecedented selectivity to parallel-stranded G-quadruplexes with exposed ends and four medium grooves. Mechanistic studies suggest that GQR associates with G-quadruplex grooves close to the end of the tetrad core, which may explain the dye's specificity to only a subset of parallel structures. This specific recognition favours the disaggregation of GQR in aqueous solutions thereby recovering the inherent fluorescence of the dye. Due to its unique features, GQR represents a valuable tool for basic biological research and the rapid discovery of novel, specific ligands that target similar structural features of G-quadruplexes.

$\mathrm{N}$ ucleic acid sequences rich in guanines $(\mathrm{G})$ have a propensity to arrange into G-quadruplexes $(\mathrm{G} 4)^{1}$. These non-canonical structures have been implicated in transcription regulation and telomere maintenance; hence, they are targeted for cancer treatment and detection ${ }^{2,3}$. Conversely, engineered G4 oligonucleotides can act as drug delivery vehicles ${ }^{4-6}$, materials for nanodevices ${ }^{7,8}$, support catalysts ${ }^{9}$ or even drugs for cancer, HIV and other diseases ${ }^{10}$. For these reasons, there is strong impetus to develop probes to improve our understanding of G4 and their ligand binding characteristics ${ }^{11}$.

Studies suggest that G4 structures are highly polymorphic and exhibit distinct differences between each other ${ }^{12,13}$. These myriad of topologies arises from the combination of several well-defined structural elements such as nucleic acid type (DNA or RNA), molecularity (monomer, dimer or tetramer), strand orientation (parallel, antiparallel or hybrid), loops (orientation, sequence and length), grooves type (narrow, wide and/or medium), end-cap morphology and number of G-tetrads ${ }^{1,14}$. Efficient probing of these motifs provides opportunities for discrimination and is also a prerequisite to the discovery and study of new G4 specific ligands ${ }^{15,16}$. We were thus motivated to develop fluorescent probes that are specific for these fundamental elements.

Several fluorescent probes capable of discerning G4 from duplex DNA have been developed. These emissive probes are generally planar structures which achieve selectivity through end-stacking with G-tetrads ${ }^{17-19}$. In contrast, structural specific G4 probes are considerably rarer. Moreover, to achieve higher specificity in distinguishing different G4s, simultaneous association with multiple structural elements is desired ${ }^{19}$. Reported probes are conceived from ligands which have been found to interact with the grooves of G4 a priori $i^{20-22}$. Therefore, such rational design strategies can have limited efficiency for discovering new G4 probes with novel selectivity ${ }^{21}$. Conversely, employing the diversity-oriented fluorescent library approach (DOFLA) is a more effective strateg $^{23-25}$; screening of diversity oriented fluorescent libraries (DOFL) can accelerate the identification of fluorescent probes with the desired qualities without former insight on the structural guidelines needed.

\section{Results}

Sensor discovery. A collection of 5000 potential fluorescent sensors were thus gathered and an unbiased, high throughput screening was performed to uncover primary hits responding to G4 (Fig. S1). Additional modifications to improve the quantum yield of the hit after binding led to the discovery of a sensitive and highly selective fluorescent sensor for G4 (GQR, Fig. 1). In the presence of 93del - an interlocked, dimeric, 
<smiles>[TeH]</smiles>

$\mathbf{R}=$<smiles>[R]OC(=O)NC(C)NC(=O)N(C(C)C)C(C)C</smiles>

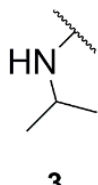

3

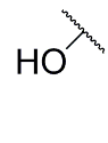

4

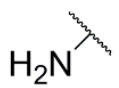

5
Figure $1 \mid$ The structures of GQR and its analogues.

parallel-stranded G4 with 4 medium grooves ${ }^{26}$ - GQR displayed up to 30 -fold increase in fluorescence at $597 \mathrm{~nm}$ and a $12 \mathrm{~nm}$ bathochromic shift in emission maximum (Fig. 2). A Job plot analysis of the GQR-93del complex revealed a binding stoichiometry of $1: 1$ (Fig. S2); the dissociation constant $\left(\mathrm{K}_{\mathrm{d}}\right)$ was thus determined as $25.18 \pm 0.02 \mu \mathrm{M}$ (Fig. 2, inset and Table S1).

Selectivity of GQR. To investigate the uniqueness of GQR, we examined its selectivity towards various G4 oligonucleotides with different structural elements (Table 1). Like 93del, J19, T95, T95 and T95-2T form parallel-stranded G4 with exposed ends and four medium grooves (Fig. S3) ${ }^{26-29}$. When incubated with GQR, all four sequences elicited a fluorescence increase from the dye (Fig. 3). Specifically, the enhancement was most pronounced with 93del. In contrast, both $c$-kit1 and Pu24T are parallel-stranded but they possess additional snapback motifs that cap the G-tetrad core ${ }^{30,31}$. Interestingly, both sequences returned negligible fluorescence when mixed with GQR (Fig. 3 and Fig. S4). On the contrary, Oxy and HT adopt non-parallel-stranded conformations (Fig. S3) ${ }^{32,33}$; GQR similarly remained quenched when incubated with either G4 (Fig. 3 and Fig. S4). Likewise, GQR remained virtually nonfluorescent in the presence of other conventional nucleic acids (Fig. 3 and Fig. S4). Previous reports of parallel-stranded selective G4 sensors generally do not display selectivity for additional motifs within the parallel-stranded structures ${ }^{20,22,34}$. To the best of our knowledge, GQR is the first fluorescent dye that is able to discern among various types of parallel-stranded G4.

Mechanism studies. Disaggregation-induced emission of GQR. Following experiments were aimed at understanding the sensing mechanism of GQR. First, we examined the photophysical

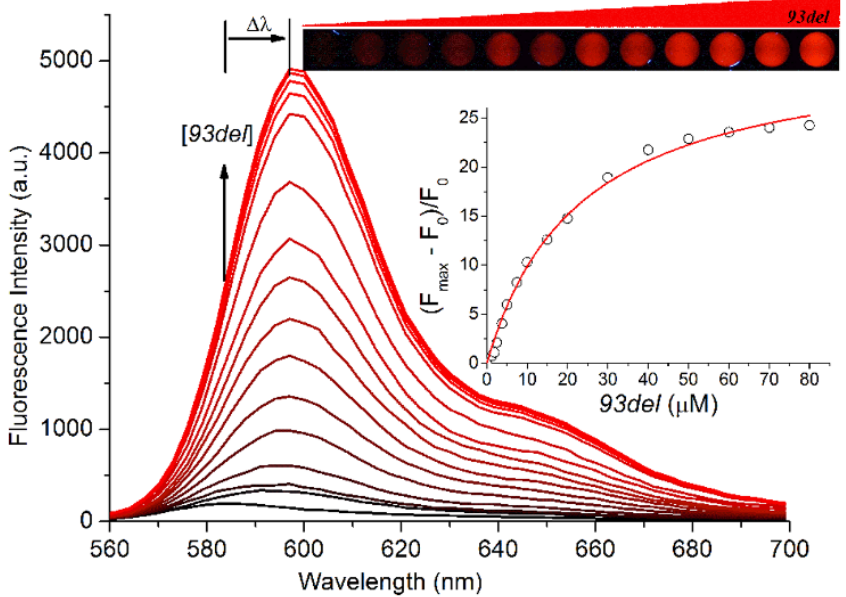

Figure $2 \mid$ GQR reacts with 93del to give a turn-on fluorescence response. Fluorescence spectra of GQR $(10 \mu \mathrm{M})$ upon incubation with serial dilutions of $93 \mathrm{del}$ (from $0-80 \mu \mathrm{M}$ ) in buffer $\left(20 \mathrm{mM} \mathrm{K}_{2} \mathrm{HPO}_{4} / \mathrm{KH}_{2} \mathrm{PO}_{4}\right.$, $100 \mathrm{mM} \mathrm{KCl}, \mathrm{pH} 7.0) . \lambda_{\mathrm{ex}}: 360 \mathrm{~nm} . \Phi_{\mathrm{F}}($ without $93 \mathrm{del})=0.014, \Phi_{\mathrm{F}}($ in $40 \mu \mathrm{M} 93 \mathrm{del})=0.28$. Inset: photographic image of GQR $(10 \mu \mathrm{M})$ in the presence of $93 \mathrm{del}$ (from $0-40 \mu \mathrm{M}$ ) and data plot of fluorescent emission intensity upon addition of $93 \mathrm{del}$ (from $0-80 \mu \mathrm{M}$ ). $\mathrm{F}_{0}$ and $\mathrm{F}_{\max }$ are the fluorescent maximum intensities of the GQR in the absence and presence of 93del respectively. $\mathrm{K}_{\mathrm{d}}=25.18 \pm 0.02 \mu \mathrm{M}$ (one site specific binding model). Values are represented as means $(n=3)$. Measurements were taken at room temperature (RT).

properties of GQR. GQR exhibited strong fluorescence emission in organic solvents, but only negligible emission in buffer (Table 2, entries 13-16 vs. entry 1). Further measurements of the absorption spectra showed significant peak broadening and a bathochromic shifts in buffer (Fig. 4a). With higher concentrations of GQR, more pronounced red-shifts were observed suggesting that this phenomenon may be a result of dye aggregation (Fig. S5). Transmission electron microscope and dynamic light scattering analysis revealed the existence of GQR-aggregates with sizes related to the dye concentration (Fig. S6 and S7). Together, these results indicate that the low fluorescence of GQR in buffer is a result of aggregation-caused quenching (ACQ) ${ }^{35}$ thereby affirming the potential of GQR to behave as fluorescent turn-on sensors for $\mathrm{G} 4^{22,36,37}$. To confirm this potential, the absorption spectra of GQR with 93del were studied. Indeed, when GQR was mixed with increasing concentrations of the G4, the spectral shape gradually shifted towards that similar to organic solvents (disaggregated state) (Fig. 4b).

Environmental sensitivity of $G Q R$. To investigate the contribution of environment polarity to the emission of GQR, we examined its emis-

\begin{tabular}{|llll}
\hline \multicolumn{1}{|l}{ Table 1 | Oligonucleotide sequences used in this work } & & \\
Name & \multicolumn{1}{c}{ Sequence $\left(5^{\prime} \rightarrow 3^{\prime}\right)$} & \multicolumn{1}{l}{ Structural Elements } & Origin \\
\hline 93del & GGGG TGGG AGGA GGGT & parallel, interlocked dimeric & Aptamer, HIV-1 integrase \\
J19 & GIGT GGGT GGGT GGGT & Aptamer, HIV-1 integrase \\
T95 & GGGT GGGT GGGT GGGT & parallel, dimeric & Aptamer, HIV-1 integrase \\
T95-2T & TT GGGT GGGT GGGT GGGT & parallel, dimeric & Aptamer, HIV-1 integrase \\
c-kit1 & AGGG AGGG CGCT GGGA GGAG GG & parallel, monomeric & c-kit oncogene promoter \\
Pu24T & TGAG GGTG GTGA GGGT GGGG AAGG & snap-back parallel & c-myconcogene promoter \\
Oxy & GGGG TTTT GGGG & antiparallel & Oxytrichanova telomere \\
HT & TT GGGTTA GGGTTA GGGTTA GGGA & hybrid & Human telomere \\
dsDNA & Sigma-D8515 & genomic dsDNA & Calf thymus \\
ssDNA & Sigma-D8899 & genomic ssDNA & Calf thymus \\
RNA & Sigma-R7250 & single-stranded & Calf liver
\end{tabular}



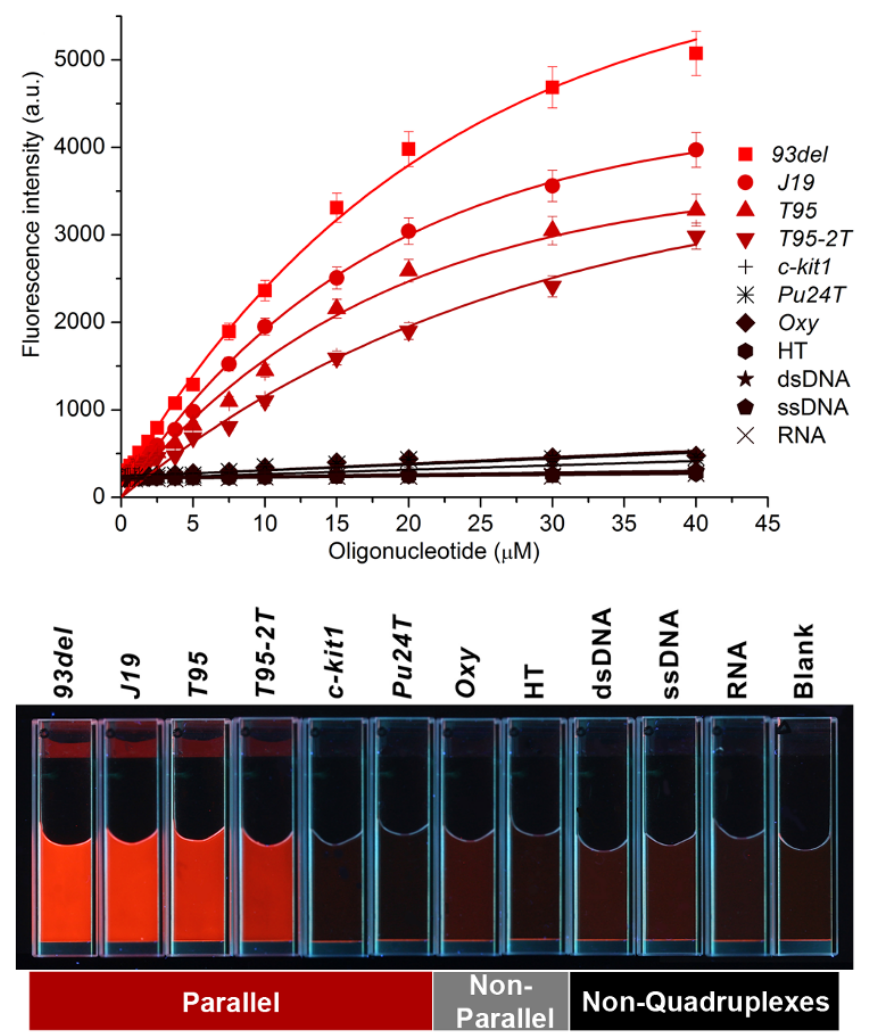

Figure 3 Selectivity of GQR to oligonucleotides. (a) Fluorescence titration of GQR with various G-quadruplexes, DNA and RNA. Conditions: GQR $(10 \mu \mathrm{M})$, nucleic acid (from 0 to $40 \mu \mathrm{M})$, buffer $\left(20 \mathrm{mM} \mathrm{K}_{2} \mathrm{HPO}_{4} / \mathrm{KH}_{2} \mathrm{PO}_{4}, 100 \mathrm{mM} \mathrm{KCl}, \mathrm{pH} 7.0\right)$, RT. $\lambda_{\text {ex }}: 360 \mathrm{~nm}, \lambda_{\text {em }}$ : $600 \mathrm{~nm}$. (b) Photographic image of GQR $(10 \mu \mathrm{M})$ mixed with various nucleic acids $(40 \mu \mathrm{M})$. Irradiation with a hand-held UV lamp at $365 \mathrm{~nm}$.

sion spectra in various toluene-methanol mixtures. We observed that the emission maximum of GQR red-shifted from 582 to $590 \mathrm{~nm}$ as solvent polarity decreases from methanol to toluene (Fig. S8). These observations suggests that the bathochromic shift in the emission of GQR when incubated with 93del is a consequence of the G4 providing a hydrophobic binding pocket for GQR thereby affirming the interaction between the two entities.

Binding mode studies. We were further interested in the interaction of GQR with its respective G4s at the molecular level. To this end, four additional analogues of GQR were studied in greater detail (Fig. 1). Like GQR, compounds 2, 3, 4 and 5 shared the same absorbance spectra with GQR in DMSO (Fig. S9) and displayed high quantum yields (Table 2, entries 17-20). However, in buffer, only 2 and 3 showed aggregation-induced absorption spectra (Fig. S9) and quenched quantum yields (Table 2, entries 21-24) analogous to GQR. Similarly, when incubated with 93del, only 2 and 3 similarly exhibited a distinct enhancement in fluorescence albeit to a lesser extent than GQR (Table 2, entries 25-28 vs. 6). These results validate the importance of disaggregation and the carboxamide group of GQR in achieving effective fluorescence probing of G4.

Molecular docking was subsequently performed to understand the binding mode ${ }^{38,39}$. As depicted in Fig. 5a, binding simulation suggests that GQR associates with the groove region of the 93del (PDB ID: $1 Y 8 D)$ core close to the ends. In contrast, a simulation of an endstacking mode revealed a dramatically weaker affinity and higher energy compared to a groove binding mode (Table S2). This result is consistent with the general lack of peak shifts in the ${ }^{1} \mathrm{H}$ imino proton NMR spectra of 93del and T95-2T upon addition of GQR an observation that contrasts that of end-stacking ligands (Fig.

\begin{tabular}{|c|c|c|c|c|}
\hline Entry & Probe & Solvent & Analyte $^{a}$ & $\Phi_{\mathrm{F}}^{\mathrm{b}}$ \\
\hline 1 & GQR & Buffer ${ }^{c}$ & - & 0.014 \\
\hline 2 & GQR & Buffer & 93del & 0.28 \\
\hline 3 & GQR & Buffer & $J 19$ & 0.22 \\
\hline 4 & GQR & Buffer & T95 & 0.19 \\
\hline 5 & GQR & Buffer & $T 95-2 T$ & 0.14 \\
\hline 6 & GQR & Buffer & $c-k i t 1$ & 0.009 \\
\hline 7 & GQR & Buffer & Pu24T & 0.005 \\
\hline 8 & GQR & Buffer & Oxy & 0.027 \\
\hline 9 & GQR & Buffer & $H T^{\prime}$ & 0.007 \\
\hline 10 & GQR & Buffer & dsDNA & 0.011 \\
\hline 11 & GQR & Buffer & ssDNA & 0.008 \\
\hline 12 & GQR & Buffer & RNA & 0.016 \\
\hline 13 & GQR & DMSO & - & 0.55 \\
\hline 14 & GQR & $\mathrm{MeOH}$ & - & 0.57 \\
\hline 15 & GQR & PEG-400 & - & 0.46 \\
\hline 16 & GQR & Tolvene & - & 0.30 \\
\hline 17 & 2 & DMSO & - & 0.30 \\
\hline 18 & 3 & DMSO & - & 0.36 \\
\hline 19 & 4 & DMSO & - & 0.39 \\
\hline 20 & 5 & DMSO & - & 0.21 \\
\hline 21 & 2 & Buffer & - & 0.013 \\
\hline 22 & 3 & Buffer & - & 0.030 \\
\hline 23 & 4 & Buffer & - & 0.33 \\
\hline 24 & 5 & Buffer & - & 0.24 \\
\hline 25 & 2 & Buffer & 93del & 0.20 \\
\hline 26 & 3 & Buffer & 93del & 0.21 \\
\hline 27 & 4 & Buffer & 93del & 0.34 \\
\hline 28 & 5 & Buffer & 93del & 0.23 \\
\hline
\end{tabular}

S10 $)^{15,17,18}$. A closer examination of the binding model suggests plausible hydrogen bonds with the carboxamide group which may account for the enhanced fluorescence of GQR compared to 2 and 3 (Fig. 5b).

\section{Discussion}

Common BODIPY dyes have a tendency to aggregate which results in self-quenching ${ }^{40,41}$. This ACQ effect often limits the label-to-analyte ratio and narrows the practical applications ${ }^{42}$. However, BODIPY can recover its high fluorescence in response to a target molecule through a recognition-induced disassembly of the aggregates. In this study, GQR-aggregates exhibit remarkable fluorescence enhancement in the presence 93del. However, water-soluble analogues show no such response under the same conditions. The discovery of GQR as an effective fluorescent sensor demonstrates that the conventional ACQ problem can, in reality, be a general mechanism for probe development ${ }^{43,44}$.

The structural polymorphism of G-quadruplexes has been supported by NMR and X-ray crystallography. Nonetheless, a simple method to distinguish different G-quadruplex structures conveniently and sensitively is highly desirable. The use of specific fluorescent probes, such as GQR, is one such strategy. Fluorescent probes can be potentially employed for mapping and tracking G-quadruplexes-based structure motifs ${ }^{17,45}$ and can also be used for rapid discovery of novel, specific anticancer drugs that recognize similar G-quadruplex structural elements to the probe.

In summary, we have described the systematic discovery of a novel fluorescent dye - GQR - which specifically "light-up" when bound to parallel G4 with end exposed medium grooves. Specific recognition and favourable binding to G4 induces the disaggregation of GQR which results in recovery of the inherent fluorescence of the dye. Preliminary mechanistic studies suggest a groove-binding mode close to the end of the quadruplex core, thereby accounting for the 

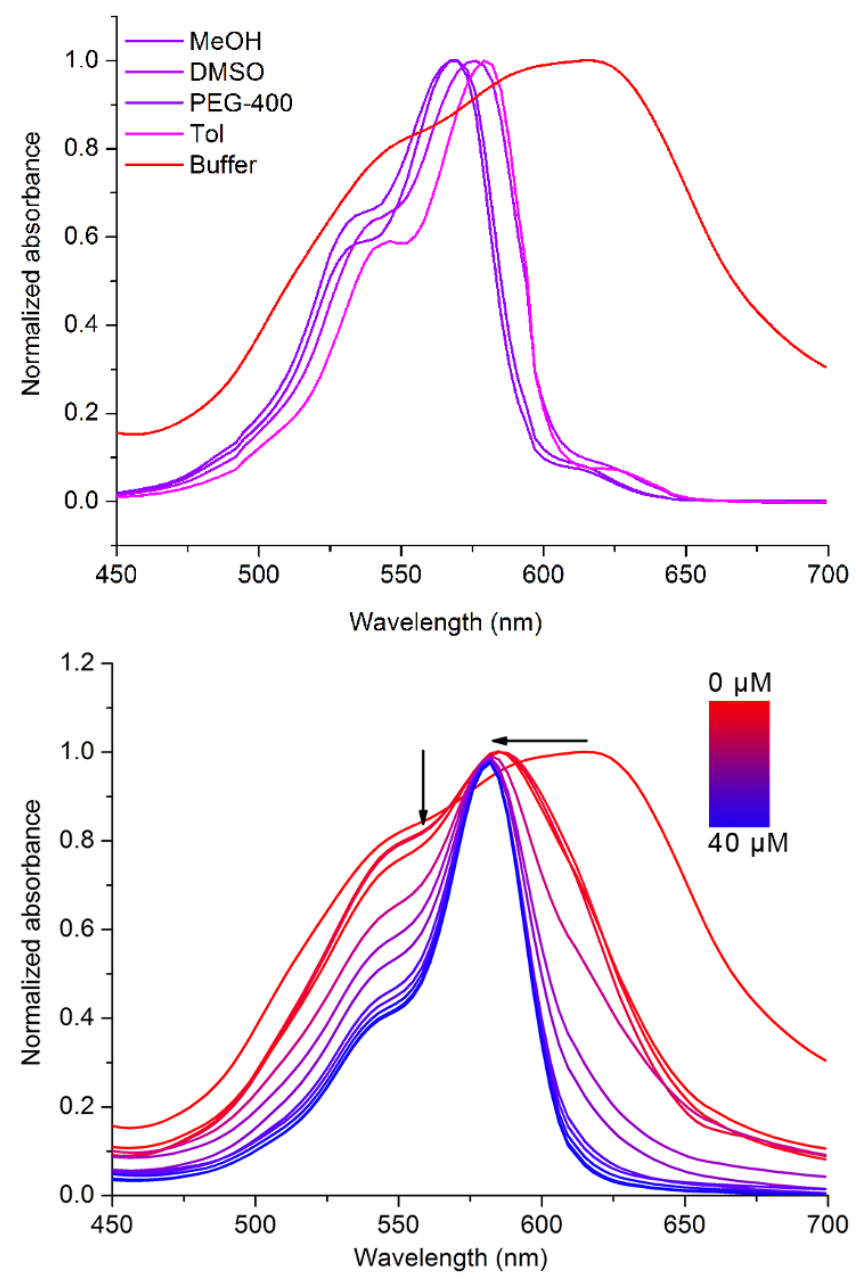

Figure $4 \mid$ Absorption spectra of GQR. (a) GQR (10 $\mu \mathrm{M})$ in various organic solvents. (b) GQR $(10 \mu \mathrm{M})$ upon incubation with serial dilutions of $93 \mathrm{del}$ (from $0-40 \mu \mathrm{M})$ in buffer. Values are represented as means $(\mathrm{n}=$ 3). Measurements were taken at RT.

high specificity. Therefore, GQR represents a promising tool for the future discovery of similar G4 groove binding ligands that can be important for disease studies, G4-based drug deliveries and nanotechnology.

\section{Methods}

Probe synthesis. Detailed description of the synthesis of each probe can be found in the Supplementary Information. Each step was characterized by high-resolution mass spectra, ${ }^{1} \mathrm{H}$ and ${ }^{13} \mathrm{C}$ NMR.

Nucleic acids. DNA oligonucleotides were synthesized and structurally verified by NMR spectroscopy as previously reported ${ }^{26-33}$. dsDNA, ssDNA and RNA were purchased from Sigma Aldrich (product number D8515, D8899 and D7250 respectively) and used as such. The nucleic acids were dissolved in buffer $(20 \mathrm{mM}$ $\mathrm{K}_{2} \mathrm{HPO}_{4} / \mathrm{KH}_{2} \mathrm{PO}_{4} 100 \mathrm{mM} \mathrm{KCl}$, pH 7.0). DNA concentration was expressed in strand molarity using a nearest-neighbor approximation for the absorption coefficients of the unfolded species.

Absorbance. UV/Vis absorption spectra of dyes and nucleic acids in buffer $(20 \mathrm{mM}$ $\mathrm{K}_{2} \mathrm{HPO}_{4} / \mathrm{KH}_{2} \mathrm{PO}_{4}, 100 \mathrm{mM} \mathrm{KCl}, \mathrm{pH}$ 7.0) were recorded from 200 to $700 \mathrm{~nm}$ using a SpectraMax M2 spectrophotometer.

Fluorescence. Fluorescence measurements were carried out on a SpectraMax M2 spectrophotometer in 96-well plates by scanning the emission spectra between 540 and $700 \mathrm{~nm}\left(\lambda_{\mathrm{ex}}=360 \mathrm{~nm}\right)$. All experiments were repeated three times. Data analysis was performed using Origin 8.0 (OriginLab Corporation, MA).

Quantum yield measurements. Quantum yields were calculated by measuring the integrated emission area of the fluorescent spectra in its respective solvents and comparing to the area measured for Coumarin 1 (reference) $(10 \mu \mathrm{M}), \Phi_{\mathrm{F}}=0.73$ in
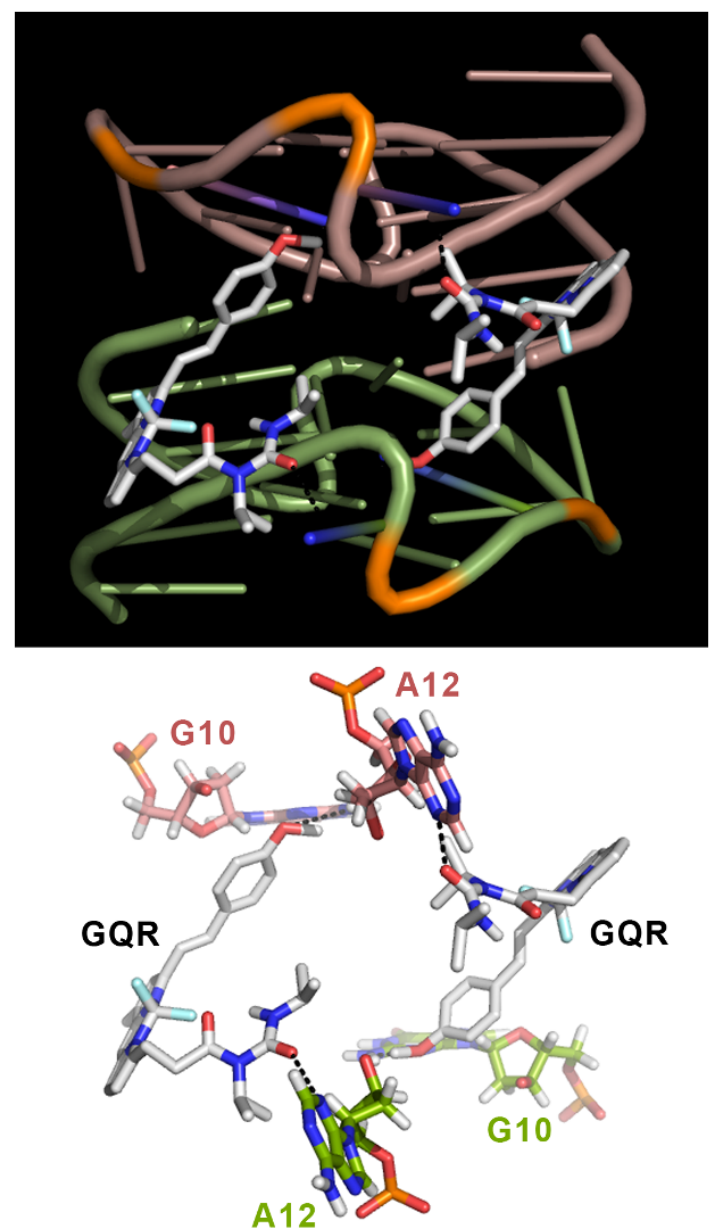

Figure $5 \mid$ Molecular docking results of GQR binding to the 93del. Illustration of groove-binding mode of GQR with 93del residues. Carbon atoms are colored in white for GQR, pink and green 93del; oxygen and nitrogen atoms are colored in red and blue respectively. (b) Plausible hydrogen bonds between GQR and 93del residues G10 and A12 (black dashed lines). Carbon atoms are colored in white for GQR, pink and green for 93del; oxygen and nitrogen atoms are colored in red and blue respectively.

ethanol ( $\eta=1.36), \lambda_{\text {ex }}=360 \mathrm{~nm}$. Quantum yields were calculated using the equation:

$$
\Phi_{F}^{\text {sample }}=\Phi_{F}^{\text {reference }}\left(\frac{F^{\text {sample }}}{F^{\text {reference }}}\right)\left(\frac{\eta^{\text {sample }}}{\eta^{\text {reference }}}\right)^{2}\left(\frac{A b s^{\text {reference }}}{A b s^{\text {sample }}}\right)
$$

where $F$ represents the area of fluorescent emission, $\eta$ is the refractive index of the solvent, and Abs is absorbance at the excitation wavelength. Emission was integrated between 540 and $700 \mathrm{~nm}$.

Dissociation constant measurements. The $\mathrm{K}_{\mathrm{d}}$ of GQR to the respective $\mathrm{G}$ quadruplexes were analyzed by Origin 8.0 (OriginLab Corporation, MA) using the following equation for a one site specific binding model:

$$
y=\frac{y_{\max } x}{K_{d}+x}
$$

Where $y$ represents the fluorescence fold change of GQR, $y_{\max }$ the fluorescence fold change of GQR when saturated with G-quadruplex and $x$ the concentration of the Gquadruplex in $\mu \mathrm{M}$.

Transmission electron microscope. GQR $(10 \mu \mathrm{M})$ was first prepared in buffer and deposited on a thin copper-support film, followed by drying in vacuo. Images of the samples were obtained with JEOL JEM 3010 HRTEM microscope and operated at $100 \mathrm{kV}$ without any contrast agent.

Dynamic light scattering. The dynamic light scattering of different concentration $\mathrm{GQR}$ and other compounds were measured at $25^{\circ} \mathrm{C}$ in buffer using quartz cell. All measurements were performed in triplicate in Zetasizer Nano ZS. 
Nuclear magnetic resonance spectroscopy. Titration with GQR was performed on a $600 \mathrm{MHz}$ NMR Bruker spectrometers equipped with a cryoprobe at $25^{\circ} \mathrm{C}$ Oligonucleotides $(200 \mu \mathrm{M})$ were dissolved in buffer $\left(20 \mathrm{mM} \mathrm{K}_{2} \mathrm{HPO}_{4} / \mathrm{KH}_{2} \mathrm{PO}_{4}\right.$, $70 \mathrm{mM} \mathrm{KCl}, \mathrm{D}_{2} \mathrm{O} / \mathrm{H}_{2} \mathrm{O}(1: 9), \mathrm{pH}$ 7.0). The spectra were recorded immediately after each addition of GQR. Water suppression was achieved using excitation sculpting.

Molecular modeling. The coordinates of 93del structures were retrieved from the Protein Data Bank (ID code 1Y8D). DNA structures were prepared for docking. The GQR structure was optimized using the Gaussian03 program (B3LYP/6-31G* level). By using Autodock 4.0, docking studies were carried out with the Lamarckian genetic algorithm following the procedure developed for G-quadruplex DNA and ligand docking ${ }^{38,39}$. Two rounds of simulation were performed. In the first round, simulated annealing was used to find a rough binding mode of GQR with 50 runs while keeping all other parameters default. The search space was subsequently reduced and another 200 runs were conducted to get a more precise result. Following the docking studies of GQR with 93del quadruplex DNA, all the figures were rendered using PyMOL v0.99.

1. Parkinson, G. N. [Fundamentals of quadruplex structures] Quadruplex nucleic acids [Neidel, S. \& Balasubramanian, S. (ed.)] [1-30] (Royal Society of Chemistry, Cambridge, 2006).

2. Folini, M., Venturini, L., Cimino-Reale, G. \& Zaffaroni, N. Telomeres as targets for anticancer therapies. Expert Opin. Ther. Targets 15, 579-593 (2011).

3. Yang, D. \& Okamoto, K. Structural insights into G-quadruplexes: towards new anticancer drugs. Future Med. Chem. 2, 619-646 (2010)

4. Chen, C., Zhou, L., Geng, J., Ren, J. \& Qu, X. Photosensitizer-incorporated quadruplex DNA-gated nanovechicles for light-triggered, targeted dual drug delivery to cancer cells. Small 9, 2793-2800 (2013).

5. Wang, K. et al. Self-assembly of a bifunctional DNA carrier for drug delivery. Angew. Chem., Int. Ed. 50, 6098-6101 (2011).

6. Shieh, Y.-A., Yang, S.-J., Wei, M.-F. \& Shieh, M.-J. Aptamer-based tumor-targeted drug delivery for photodynamic therapy. ACS Nano 4, 1433-1442 (2010).

7. Huang, Y. C. \& Sen, D. A contractile electronic switch made of DNA. J. Am. Chem Soc. 132, 2663-2671 (2010).

8. Li, T., Wang, E. \& Dong, S. Potassium-lead-switched G-quadruplexes: a new class of DNA logic gates. J. Am. Chem. Soc. 131, 15082-15083 (2009).

9. Tang, Z., Gonçalves, D. P. N., Wieland, M., Marx, A. \& Hartig, J. S. Novel DNA catalysts based on G-quadruplex recognition. ChemBioChem 9, 1061-1064 (2008).

10. Collie, G. W. \& Parkinson, G. N. The application of DNA and RNA Gquadruplexes to therapeutic medicines. Chem. Soc. Rev. 40, 5867-5892 (2011).

11. Davis, J. T. G-quartets 40 Years later: from 5'-GMP to molecular biology and spramolecular chemistry. Angew. Chem., Int. Ed. 43, 668-698 (2004).

12. Neidle, S. The structures of quadruplex nucleic acids and their drug complexes. Curr. Opin. Struct. Biol. 19, 239-250 (2009).

13. Burge, S., Parkinson, G. N., Hazel, P., Todd, A. K. \& Neidle, S. Quadruplex DNA: sequence, topology and structure. Nucleic Acids Res. 34, 5402-5415 (2006).

14. Phan, A. T., Kuryavyi, V. \& Patel, D. J. DNA architecture: from G to Z. Curr. Opin. Struct. Biol. 16, 288-298 (2006).

15. Largy, E., Granzhan, A., Hamon, F., Verga, D. \& Teulade-Fichou, M.-P. Visualizing the quadruplex: from fluorescent ligands to light-up probes. Top. Curr. Chem. 330, 111-177 (2013).

16. Gai, W. et al. A dual-site simultaneous binding mode in the interaction between parallel-stranded G-quadruplex [d(TGGGGT)]4 and cyanine dye 2,2' -diethyl-9methyl-selenacarbocyanine bromide. Nucleic Acids Res. 41, 2709-2722 (2013).

17. Vummidi, B. R., Alzeer, J. \& Luedtke, N. W. Fluorescent probes for G-quadruplex structures. ChemBioChem 14, 540-558 (2013).

18. Ma, D.-L., Shiu-Hin Chan, D., Yang, H., He, H.-Z. \& Leung, C.-H. Luminescent Gquadruplex probes. Curr. Pharm. Des. 18, 2058-2075 (2012).

19. Chang, C.-C. et al. Detection of quadruplex DNA structures in human telomeres by a fluorescent carbazole derivative. Anal. Chem. 76, 4490-4494 (2004).

20. Nikan, M., Di Antonio, M., Abecassis, K., McLuckie, K. \& Balasubramanian, S. An acetylene-bridged 6,8-purine dimer as a fluorescent switch-on probe for parallel G-quadruplexes. Angew. Chem., Int. Ed. 52, 1428-1431 (2013).

21. Largy, E., Saettel, N., Hamon, F., Dubruille, S. \& Teulade-Fichou, M.-P. Screening of a chemical library by HT-G4-FID for discovery of selective G-quadruplex binders. Curr. Pharm. Des. 18, 1992-2001 (2012).

22. Li, T., Wang, E. K. \& Dong, S. J. Parallel G-quadruplex-specific fluorescent probe for monitoring DNA structural changes and label-free detection of potassium ion. Anal. Chem. 82, 7576-7580 (2010).

23. Vendrell, M., Zhai, D., Er, J. C. \& Chang, Y.-T. Combinatorial strategies in fluorescent probe development. Chem. Rev. 112, 4391-4420 (2012).

24. Kang, N. Y., Ha, H. H., Yun, S. W., Yu, Y. H. \& Chang, Y. T. Diversity-driven chemical probe development for biomolecules: beyond hypothesis-driven approach. Chem. Soc. Rev. 40, 3613-3626 (2011).

25. Vendrell, M., Lee, J.-S. \& Chang, Y.-T. Diversity-oriented fluorescence library approaches for probe discovery and development. Curr. Opin. Chem. Biol. 14, 383-389 (2010).

26. Phan, A. T. et al. An interlocked dimeric parallel-stranded DNA quadruplex: A potent inhibitor of HIV-1 integrase. Proc. Natl. Acad. Sci. 102, 634-639 (2005).
27. Kelley, S., Boroda, S., Musier-Forsyth, K. \& Kankia, B. I. HIV-integrase aptame folds into a parallel quadruplex: A thermodynamic study. Biophys. Chem. 155, 82-88 (2011).

28. Do, N. Q. \& Phan, A. T. Monomer-dimer equilibrium for the $5^{\prime}-5^{\prime}$ Stacking of propeller-type parallel-stranded G-quadruplexes: NMR structural study. Chem.Eur. J. 18, 14752-14759 (2012)

29. Do, N. Q., Lim, K. W., Teo, M. H., Heddi, B. \& Phan, A. T. Stacking of Gquadruplexes: NMR structure of a G-rich oligonucleotide with potential anti-HIV and anticancer activity. Nucleic Acids Res. 39, 9448-9457 (2011).

30. Phan, A. T., Kuryavyi, V., Burge, S., Neidle, S. \& Patel, D. J. Structure of an unprecedented G-quadruplex scaffold in the human c-kit promoter. J. Am. Chem. Soc. 129, 4386-4392 (2007)

31. Phan, A. T., Kuryavyi, V., Gaw, H. Y. \& Patel, D. J. Small-molecule interaction with a five-guanine-tract G-quadruplex structure from the human MYC promoter. Nat. Chem. Biol. 1, 167-173 (2005).

32. Marincola, F. C., Virno, A., Randazzo, A. \& Lai, A. Effect of rubidium and cesium ions on the dimeric quaduplex formed by the Oxytricha Nova telomeric repeat oligonucleotide D(GGGGTTTTGGGG). Nucleosides, Nucleotides Nucleic Acids 26, 1129-1132 (2007).

33. Luu, K. N., Phan, A. T., Kuryavyi, V., Lacroix, L. \& Patel, D. J. Structure of the human telomere in $\mathrm{K}+$ solution: an intramolecular $(3+1) \mathrm{G}$-quadruplex scaffold. J. Am. Chem. Soc. 128, 9963-9970 (2006)

34. Nicoludis, J. M., Barrett, S. P., Mergny, J.-L. \& Yatsunyk, L. A. Interaction of human telomeric DNA with N-methyl mesoporphyrin IX. Nucleic Acids Res. 40 5432-5447 (2012).

35. Hong, Y., Lam, J. W. Y. \& Tang, B. Z. Aggregation-induced emission. Chem. Soc. Rev. 40, 5361-5388 (2011).

36. Alzeer, J. \& Luedtke, N. W. pH-mediated fluorescence and G-quadruplex binding of amido phthalocyanines. Biochemistry 49, 4339-4348 (2010).

37. Yang, Q. et al. Verification of specific G-quadruplex structure by using a nove cyanine dye supramolecular assembly: I. Recognizing mixed G-quadruplex in human telomeres. Chem. Commun., 1103-1105 (2009).

38. Haider, S. \& Neidle, S. [Molecular modeling and simulation of G-quadruplexes and quadruplex-ligand complexes] G-quadruplex DNA [Baumann, P. (ed.)] [17-37] (Humana Press, New York, 2010).

39. Morris, G. M. et al. Automated docking using a Lamarckian genetic algorithm and an empirical binding free energy function. J. Comput. Chem. 19, 1639-1662 (1998).

40. Liu, H. Z. et al. A selective colorimetric and fluorometric ammonium ion sensor based on the H-aggregation of an aza-BODIPY with fused pyrazine rings. Chem. Commun. 47, 12092-12094 (2011).

41. Lu, H. et al. Specific Cu2+-induced J-aggregation and $\mathrm{Hg} 2+$-induced fluorescence enhancement based on BODIPY. Chem. Commun. 46, 3565-3567 (2010).

42. Yuan, W. Z. et al. Changing the behavior of chromophores from aggregation caused quenching to aggregation-induced emission: development of highly efficient light emitters in the solid state. Adv. Mater. 22, 2159-2163 (2010)

43. Zhai, D. et al. Development of a fluorescent sensor for an illicit date rape drug GBL. Chem. Commun. 49, 6170-6172 (2013).

44. Xu, W. et al. Make caffeine visible: a fluorescent caffeine "Traffic Light" detector Sci. Rep. 3, DOI: 10.1038/srep02255 (2013).

45. Balasubramanian, S. \& Neidle, S. G-quadruplex nucleic acids as therapeutic targets. Curr. Opin. Chem. Biol. 13, 345-353 (2009).

\section{Acknowledgments}

This study was supported by intramural funding and grant 10/1/21/19/656 from the A*STAR (Agency for Science, Technology and Research, Singapore) Biomedical Research Council. L.Z. acknowledges the financial support from Natural Science Foundation of China (No. 11105150) and the Special Financial Grant from China Postdoctoral Science Foundation (No. 2013T60613). J.C.E. acknowledges the receipt of a NGS scholarship. The authors thank Dr. Animesh Samanta and Dr. Santanu Jana for their great help in TEM analysis and discussion.

\section{Author contributions}

Y.T.C. and A.T.P. conceived the idea and directed the work. L.Z. and J.C.E. designed the experiments and co-wrote the manuscript. L.Z. designed, screened and identified the G-quadruplex sensor and analyzed the photophysical properties and binding mechanism. J.C.E. and J.Y. designed, synthesized and characterized all dye compounds used in this study. K.K.G. designed and synthesized the diversity-oriented fluorescent libraries. W.J.C. synthesized and characterized all G-quadruplex sequences. W.X. performed the DLS analysis. W.Z. performed the molecular docking studies. All authors contributed to data analysis and manuscript writing.

\section{Additional information}

Supplementary information accompanies this paper at http://www.nature.com/ scientificreports

Competing financial interests: The authors declare no competing financial interests. 
How to cite this article: Zhang, L.Y. et al. Discovery of a Structural-Element Specific G-Quadruplex "Light-Up” Probe. Sci. Rep. 4, 3776; DOI:10.1038/srep03776 (2014). cc) (1) \&) $\odot$ This work is licensed under a Creative Commons Attribution列 visit http://creativecommons.org/licenses/by-nc-nd/3.0 